\title{
Does the complex susceptibility of the Hénon map have a pole in the upper-half plane ? A numerical investigation.
}

\author{
B. Cessac \\ INRIA, 2004 Route des Lucioles, 06902 Sophia-Antipolis, France. \\ Institut Non Linaire de Nice, 1361 route des Lucioles, 06560 Valbonne, France. \\ Université de Nice, Parc Valrose, 06000 Nice, France.
}

\begin{abstract}
It has been rigorously shown in [18] that the complex susceptibility of chaotic maps of the interval can have a pole in the upper-half complex plane. We develop a numerical procedure allowing to exhibit this pole from time series. We then apply the same analysis to the Hénon map and conjecture that the complex susceptibility has also a pole in the upper-half complex plane.
\end{abstract}

PACS numbers: 02.70.-c,05.10.-a,05.45.-a

Submitted to: Nonlinearity 
Consider a dynamical system $f: \mathcal{M} \rightarrow \mathcal{M}$, on a manifold $\mathcal{M}$ endowed with the Lebesgue measure. Assume that there is a unique $f$-ergodic probability measure $\rho$ so that $\lim _{T \rightarrow \infty} \frac{1}{T} \sum_{t=1}^{T} \phi\left(f^{n}(x)\right)=\int \phi d \rho$ for continuous functions $\phi$ and for Lebesguealmost every $x \in \mathcal{M}$. Then, $\rho$ is called the SRB measure or physical measure (see 24] for a discussion on these two terminologies). Assume that when $f$ is perturbed in its neighborhood, the average value $\rho(A)$ of a given smooth observable $A$ varies differentiably with $f$. Then the derivative operator is called the linear response function. In the case of uniformly hyperbolic systems, and provided that $f$ and its perturbations are sufficiently smooth, then the linear response is given by [19]:

$$
\delta \rho(A)=\sum_{n=0}^{\infty} \rho\left(X . \nabla\left(A \circ f^{n}\right)\right),
$$

where $X$ is a (time-independent) perturbation. Ruelle has indeed shown that this series is convergent for suitable perturbations. In the case of a time-dependent perturbation $X_{t}$ this formula extends formally to:

$$
\delta \rho_{t}(A)=\sum_{n=0}^{\infty}\left(\kappa_{n} X_{t-n}\right) A .
$$

The operator $\kappa_{n}: \mathcal{H} \rightarrow \mathcal{B}^{*}$, where $\mathcal{H}$ is a suitable space of vector fields and $\mathcal{B}^{*}$ the dual of a suitable space of functions, is defined by:

$$
\left(\kappa_{n} X_{t-n}\right) A=\rho\left(X_{t-n} . \nabla\left(A \circ f^{n}\right)\right) ; \quad n \geq 0,
$$

while, for $n<0,\left(\kappa_{n} X_{t-n}\right) A=0$ (causality). It is called the "response function". In this case, one recovers the standard definition of the linear response in non equilibrium statistical mechanics ((2) is a convolution product), and classical results such as fluctuation-dissipation relations or Onsager theory can be recovered [19]. Moreover, this result does not require the assumption of closeness to equilibrium.

However, one can find examples of non uniformly hyperbolic systems where the series (11) does not converge, even for maps of the interval with absolutely continuous invariant measures [18]. In this case, one considers the complex susceptibility function, $\chi(\omega)=\left(\hat{\kappa}_{\omega} X\right) A$ where $\hat{\kappa}_{\omega}$ is the Fourier transform of the response function. It is also convenient to use the variable $\lambda=e^{i \omega}$. Then, $\chi(\omega)=\Psi(\lambda)$. Under some conditions on $X$ and $f$ (interval maps), Ruelle proved that $\Psi(\lambda)$ extends to a meromorphic extension with no pole at $\lambda=1$. He also showed that this function has a pole inside the unit disk $|\lambda|<1$ (i.e. in the upper-half complex plane for the frequencies $\omega$ ) and other poles, corresponding to the eigenvalues of the Perron-Frobenius operator (RuellePollicott resonances [12, 14]), outside the unit disk (in the lower-half complex plane). The existence of a pole of the complex susceptibility in the upper-half complex plane must not be misinterpreted. This is not a "violation of causality", which has no meaning in a dynamical system, causal by construction. This simply expresses that an arbitrary small perturbation of $f$ does not give a response proportional to the perturbation. According to Ruelle: "this might be expressed by saying that $\rho$ is not linearly stable". 
This result is quite interesting and intriguing for a physicist and two natural questions arise. Does this property exist in larger dimensional chaotic dynamical systems ? And is it possible to have an experimental/numerical characterization of the effects of this pole? The first question is difficult to address from a mathematical point of view and is still unresolved even for classical examples such as the Hénon map. The second one is the main concern of the present paper. We indeed propose a numerical experimentation protocol allowing to numerically approximate the complex susceptibility (7) and to investigate the numerically observable effects of having a pole in the upper-half complex plane.

The paper is organized as follows. After a brief summary of Ruelle's results for the logistic map (section 1), we describe our numerical methods in section 2 and apply it to the logistic map where one can use the guidelines of Ruelle's mathematical results as a validation (section 3). Then, we apply it to the Hénon map for the standard parameter values and conjecture that this map could also exhibit a pole in the upper-half complex plane (section 41).

\section{Reminder of Ruelle's results.}

The dynamical system defined by the logistic map:

$$
f(x)=1-2 x^{2}, \quad x \in[-1,1]
$$

has an absolutely continuous ergodic measure with density :

$$
\rho(x)=\frac{1}{\pi \sqrt{1-x^{2}}} .
$$

The linear response (11) reads, in this case:

$$
\delta \rho(A)=\sum_{n=0}^{\infty} \int X(x) \frac{d}{d x} A\left(f^{n} x\right) \rho(d x),
$$

while the complex susceptibility reads:

$$
\chi(\omega)=\Psi(\lambda)=\sum_{n=0}^{\infty} \lambda^{n} \int A^{\prime}\left(f^{n} x\right)\left(f^{n}\right)^{\prime}(x) X(x) \rho(d x)
$$

with $\lambda=e^{i \omega}$. Ruelle has shown the following results [18]. Consider the variable change $x=\sin \left(\frac{\pi}{2} y\right)=\varpi(y)$ which maps $f$ on $g(y)=1-2|y|$ [tent map]. Then the density $\rho$ is mapped onto the density $\sigma_{0}(y)=\frac{1}{2}$. In this variable, we have :

$$
\Psi(\lambda)=\sum_{n=0}^{\infty} \lambda^{n} \int_{-1}^{1}\left(\mathcal{L}_{0}^{n} Y\right)(s) B^{\prime}(s) d s
$$

where $B=A \circ \varpi, Y(y)=\sigma_{0}(y) \frac{X(\varpi(y))}{\varpi^{\prime}(y)}$ and $\mathcal{L}_{0} \Phi(y)=\Phi\left(\frac{y-1}{2}\right)-\Phi\left(\frac{1-y}{2}\right)$.

Consider first a square integrable holomorphic perturbation $X \equiv X_{0}$ that vanishes at $\{-1,1\}$. Then, the series (8) has a meromorphic extension in $\mathbf{C}$ and has no pole 
inside the unit disc. Moreover, it is holomorphic at $\lambda=1$ (resp. $\omega=0$ ). Indeed, one has, using integration by parts:

$$
\Psi(\lambda)=-\sum_{n=0}^{\infty} \lambda^{n} \int_{-1}^{1}\left(\mathcal{L}^{n} Y_{0}^{\prime}\right)(s) B(s) d s,
$$

where $\mathcal{L}$ is the Perron-Frobenius operator:

$$
\mathcal{L} \Phi(y)=\frac{1}{2} \Phi\left(\frac{y-1}{2}\right)+\frac{1}{2} \Phi\left(\frac{1-y}{2}\right)
$$

In the space of analytic functions, and in the basis of monomials $\left\{y^{m}\right\}, m=$ $0,1,2, \ldots$ this operator is represented by an infinite triangular matrix. The eigenvalues are on the diagonal. They are of the form $1 / 4^{n}, n=0,1 \ldots$ The corresponding right eigenfunctions are (Bernoulli) polynomials such that $\sigma_{0}(y)=\frac{1}{2}$ (invariant density corresponding to the eigenvalue 1);

$$
\sigma_{1}(y)=-\frac{1}{3}-2 y+y^{2}
$$

(eigenvalue $\frac{1}{4}$ ), etc... This simple argument can be found in [10] pp 123-125. This result is also a direct consequence of [3].

Therefore, for a perturbation $Y_{0}^{\prime}(y)=\sum_{k=1}^{\infty} C_{k} \sigma_{k}(y)$, and for an observable $A$ such that the integral $\int_{-1}^{1} Y_{0}^{\prime}(s) B(s) d s$ is finite, one finds easily that the susceptibility has poles $\lambda_{k}^{-1}, k \geq 1$, outside the unit disk. For example, if $Y_{0}^{\prime}(y)=\sigma_{1}(y)$, the series (9) reads:

$$
\Psi(\lambda)=-\sum_{n=1}^{\infty}\left(\frac{\lambda}{4}\right)^{n} \int_{-1}^{1} \sigma_{1}(s) B(s) d s
$$

This case is used as a benchmark for the numerical procedure developed in the next section. The corresponding perturbation $X_{0}$ reads, in the variable $x$ :

$$
X_{0}(x)=\pi \sqrt{1-x^{2}}\left[1-\frac{2}{3 \pi} \arcsin (x)-\frac{4}{\pi^{2}} \arcsin ^{2}(x)+\frac{8}{3 \pi^{3}} \arcsin ^{3}(x)\right]
$$

where $X_{0}( \pm 1)=0$.

Ruelle has also shown that there is a meromorphic function :

$$
\Phi_{-}(z)=\frac{1}{z+1}+w(z)
$$

where $w(z)$ is of order $O(z+1)$, and where $\Phi_{-}( \pm 1)=0$ such that $\mathcal{L}_{0} \Phi_{-}=\sqrt{f^{\prime}(-1)} \Phi_{-}$ $\left(=2 \Phi_{-}\right.$in our case). The function $w$ in (14) is therefore a solution of:

$$
w\left(\frac{z-1}{2}\right)-w\left(\frac{1-z}{2}\right)-2 w(z)=\frac{2}{3-z} .
$$

$w$ is not an analytic function. It can be obtained via the recursion:

$$
w(z)=\left\{\begin{array}{rc}
w(2 z+1)+\frac{1}{2-2 z} ; & z<0 ; \\
-w(1-2 z)-\frac{1}{2+2 z} ; & z>0
\end{array}\right.
$$


reminiscent of the Tagaki function discussed by Gaspard in the context of the multibaker map [10]. Returning to the variable $x$, the function $\Phi_{-}$corresponds to :

$$
X_{-}(x)=\pi \sqrt{1-x^{2}}\left[\frac{1}{\frac{2}{\pi} \arcsin (x)+1}+w\left(\frac{2}{\pi} \arcsin (x)+1\right)\right] .
$$

The corresponding series $\Psi_{-}(\lambda)=\sum_{n=0}^{\infty} \lambda^{n} \int \rho(x) A^{\prime}\left(f^{n} x\right)\left(f^{n}\right)^{\prime}(x) X_{-}(x) d x$, converges if $|\lambda| \sqrt{\left|f^{\prime}(-1)\right|}<1$, and, in its domain of convergence, has the form $\frac{G_{A}}{1-\lambda \mid \sqrt{\left|f^{\prime}(-1)\right|}}$ where $G_{A}$ is a constant depending on $A$. The complex susceptibility has therefore a pole at $\lambda=\frac{1}{\sqrt{f^{\prime}(-1)}}=\frac{1}{2}$, inside the unit disk (resp. $\omega=i \log (2)$ ). Therefore, the series $\Psi_{-}$diverges for real frequencies $\omega$.

\section{The numerical method.}

The numerical method used here has been introduced by Reick in [13] and independently in [7, 8, 9]. It is purely heuristic and there is no mathematical proof of its validity, though a discussion with some analytic developments can be found in [13] and [9]. Thus, in this section, we shall use formal expressions.

Consider a time-dependent perturbation of $f$ of type $t$ :

$$
x_{t+1}=f\left[x_{t}+\epsilon X_{t}\left(x_{t}\right)\right] .
$$

The variation of the average value of $A$, at time $t$, is formally given by:

$$
\Delta \rho_{t}(A)=\epsilon \sum_{n=-\infty}^{t} \int A^{\prime}\left(f^{t-n} x\right)\left(f^{t-n}\right)^{\prime}(x) X_{n}(x) \rho(d x)+O\left(\epsilon^{2}\right) .
$$

which corresponds to (2) to the linear order and up to a factor $\epsilon$. Note that the term $O\left(\epsilon^{2}\right)$ is not uniform in $t$.

Consider now perturbations of type $X_{t}(x) \equiv X(x) e^{-i \omega t}$ where $\omega$ is real. Then:

$$
\Delta \rho_{t}(A)=\epsilon e^{-i \omega t} \chi(\omega)+O\left(\epsilon^{2}\right),
$$

where $\chi(\omega)$, the complex susceptibility for $X(x)$, is given by eq. (7). Then, at the linear order in $\epsilon$ :

$$
\chi(\omega)=\frac{\Delta \rho_{t}(A) e^{i \omega t}}{\epsilon} .
$$

Since $\chi(\omega)$ does not depend on time one may write:

$$
\chi(\omega)=\frac{1}{\epsilon T} \sum_{t=1}^{T} \Delta \rho_{t}(A) e^{i \omega t}
$$

$\ddagger$ Note that this way of perturbing avoids the introduction of $f^{-1}$ in the computation of the response function as found e.g. in [19]. It allows one to write convolution formulae and the Fourier transform in the standard form they have for continuous time systems. This was the main motivation for this choice in [9] and we pursue along these lines in the present paper. 
The time-dependent SRB state $\rho_{t}$ involves an average over initial conditions in the distant past [19]. One can argue (see [13] Appendix A) that the above average over $t$ makes the average over initial conditions unnecessary. The idea is therefore to replace $\Delta \rho_{t}(A)$ by $A\left(x^{\prime}(t)\right)-A(x(t))$ where $x^{\prime}(t)$ is a typical trajectory of the perturbed system, with a perturbation $X(x) e^{-i \omega t}$ and $x(t)$ is a typical trajectory of the unperturbed system. Then, one computes:

$$
S_{T}(\omega, x)=\frac{1}{\epsilon T} \sum_{t=1}^{T}\left[A\left(x^{\prime}(t)\right)-A(x(t))\right] e^{i \omega t}
$$

The hope is that $S_{T}(\omega, x) \rightarrow \chi(\omega)$ as $T \rightarrow \infty$, where the limit is independent of $x(0)$. There is no mathematical proof of the above statement. Note that $T$ must be taken so large that roundoff errors play their role of selecting the limit to be the SRB state. On practical grounds, as discussed in [13, this procedure is numerically reliable provided that $\epsilon T$ is sufficiently large and $\omega T>>1$ (basically one uses $\epsilon T \omega>>1$ ). These conditions have been checked in the simulations performed in the present paper. Since $S_{T}(\omega, x)$ is expected to give a fairly good approximation of $\chi(\omega)$, the Fourier transform of the linear response, when $T$ is sufficiently large, one also expects that the inverse Fourier series of $S_{T}(\omega, x)$, called $R(t)$ (where we drop the $T$ and $x$ for simplicity) gives a fairly good approximation of the linear response (2).

\section{Remarks}

(i) The quantity obtained by this procedure is, stricto sensu, not the linear response but the total response since one can apply it for arbitrary large $\epsilon$. As a test, one must a posteriori check that the susceptibility is independent of $\epsilon$ on a certain range of small $\epsilon$ values [e.g. it does not vary if one replaces $\epsilon$ by $2 \epsilon$ ].

(ii) In the case of the logistic map, any typical trajectory of (4) approaches the points \pm 1 within a distance of order $\epsilon$ with a characteristic time of order $\frac{1}{\sqrt{\epsilon}}$. In a numerical simulation where $\epsilon$ is small but finite this arises often, especially if one respects the condition $\epsilon T \omega>>1$. However, in this case, a perturbation $X(x) e^{-i \omega t}$ can push the trajectory out of the interval $[-1,1]$ leading to an exponential divergence. To avoid this effect we have used a perturbation $X(x, t)=X(x)\left(1+e^{-i \omega t}\right)$ where $X$ is positive about $x=-1$ and negative about $x=+1$, so that the perturbation is always directed inside the interval $[-1,1]$ whenever $x= \pm 1$.

\section{Application to the logistic map.}

\subsection{A benchmark.}

Let us first consider the case where the complex susceptibility is well defined on the real axis (for the frequency $\omega$ ) by the series (7). More precisely, we consider a perturbation, corresponding to the Ruelle-Pollicott resonance $\frac{1}{4}$. In the variable $x$ this perturbation is given by eq. (13). For the observable $A$ one can use any choice such that the integral 
$\int_{-1}^{1} \sigma_{1}(s) B(s) d s$ in (12) is finite. We choose $A=X_{0}$. The complex susceptibility reads $\Psi(\lambda)=-\frac{C}{4-\lambda}$ where $C>0$ is a constant.

In the figure 17a the complex susceptibility, computed with this algorithm and compared to the theoretical value, is drawn. To generate the real and imaginary parts of the susceptibility we run two independent simulations. Starting from the same mother trajectory, we apply in the first case a perturbation $\epsilon \cos (\omega t)$ and compute the corresponding susceptibility, which gives $\operatorname{Re}(\chi(\omega))$. In the second case we apply a perturbation $-\epsilon \sin (\omega t)$, which gives $\operatorname{Im}(\chi(\omega))$. Note that the perturbations are therefore real and the trajectories stay on the real axis. The amplitude of the perturbation was fixed to $\epsilon=10^{-2}, 5 \times 10^{-3}, 10^{-3}$. Averages are performed by computing the time average $S_{T}(\omega, x)(23)$ for $N$ initial conditions $x_{n}, n=1 \ldots N$ randomly chosen and then computing $\frac{1}{N} \sum_{n=1}^{N} S_{T}\left(\omega, x_{n}\right)$. This procedure allows us to reduce the numerical noise and to compute error bars. We took $T=10^{6}, N=10$ for $\epsilon=10^{-2}$; $T=2.10^{6}, N=20$ for $\epsilon=5 \times 10^{-3}$ and $T=10^{7}, N=10$ for $\epsilon=10^{-3}$. Note that numerical noise is large and that the resonance curve is very flat, requiring to have small error bars. This requires an average over a very long time $T$, roughly given by the condition $\epsilon T \omega>>1$, and limits the range of $\epsilon$ values that one can reach. Note that the condition $\epsilon T \omega>>1$ is always violated as $\omega$ approaches zero, whatever the (finite) value of $T$. This explains the discrepancy observed for small frequencies. If one excludes this, the agreement is quite good. Note also that the experimental curve has a magnitude that does not depend of $\epsilon$, in this range of $\epsilon$ values, as expected.

The (approximate) linear response $R(t)$ can be easily obtained by computing the inverse Fourier series of $\chi(\omega)$ (e.g. by a direct summation). It is drawn in Figure 1b (in log scale for the $y$ axis). Note that up to numerical noise $R(t)$ is real, as expected. One observes an exponential decay very close to the theoretically expected decay $4^{-t}$, corresponding to the mixing rate given by the first Ruelle-Pollicott resonance (and also to the characteristic decay rate toward equilibrium, in agreement with the fluctuationdissipation theorem). Note that the range of validity for the interpolation is very thin $(\sim[0,4])$. Indeed, rapidly the perturbation becomes so weak that one measures only the numerical noise.

\subsection{Perturbation $X_{-}$.}

As we saw, the perturbation (17) leads to a diverging series (7) for real frequencies. However, when computing the expression (23) one is not dealing with a series, but with a finite sum, which diverges as $T$ grows. The divergence rate provides useful hints on the presence of the pole.

Consider indeed the formal expression (19) of $\Delta \rho_{t}(A)$ in the case of the logistic map.

$f^{t-n}$ has $t-n$ zeros and oscillates rapidly (for large $t-n$ ) with a period $\sim 4^{n-t}$. One can decompose the integral $\int_{-1}^{+1} \rho(x) A^{\prime}\left(f^{t-n} x\right)\left(f^{t-n}\right)^{\prime}(x) X(x) d x$ over intervals delimited 

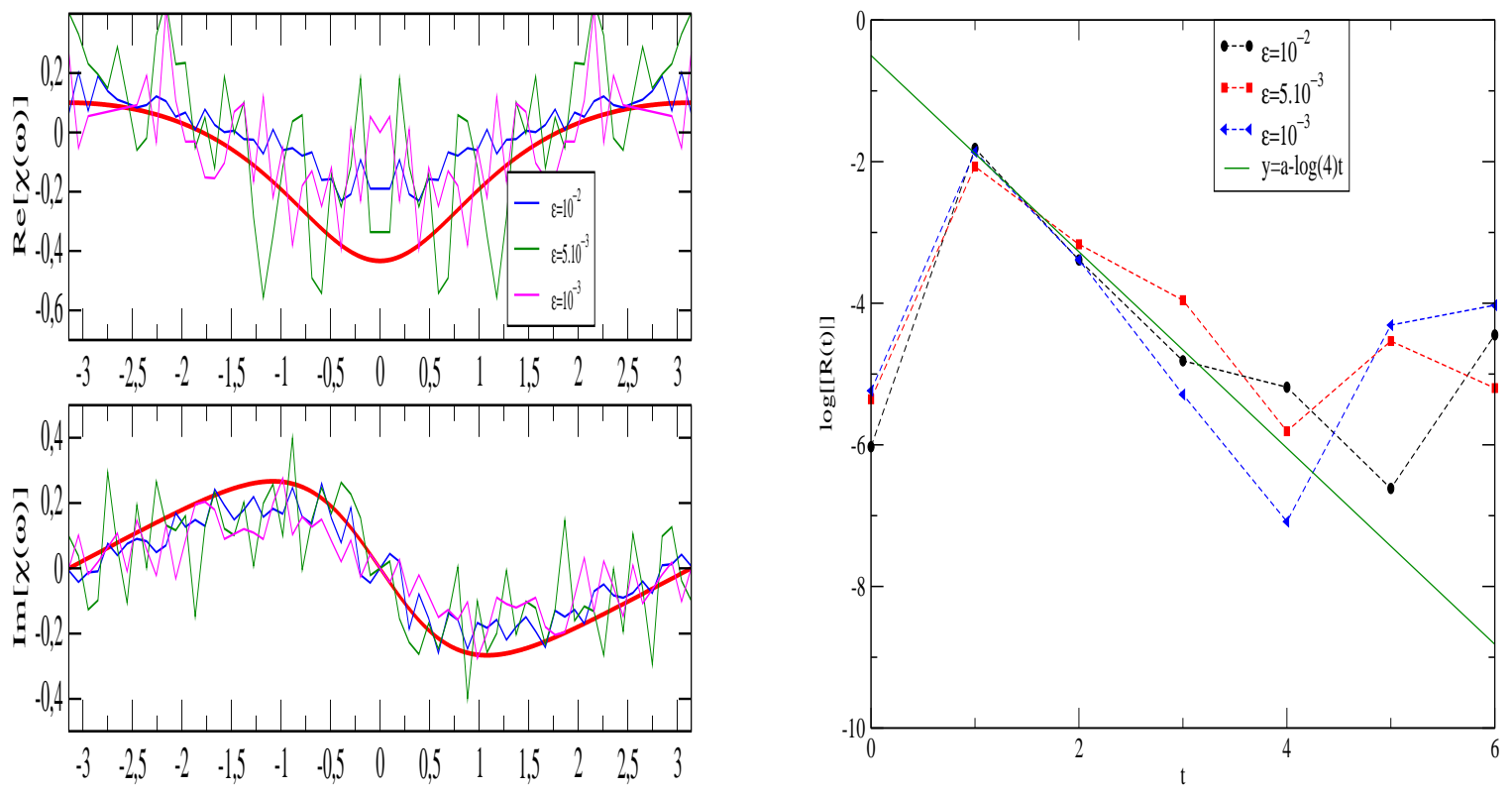

Figure 1. (a) Susceptibility for a perturbation (13) and an observable also given by (13), for $\epsilon=10^{-2}, 5 \times 10^{-3}, 10^{-3}$. (b) Corresponding approximate linear response [denoted by $\mathrm{R}(\mathrm{t})]$, in log scale and theoretically expected curve $y=a-\log (4) t$.

by the zeros of $f^{t-n}$. The density $\rho$ has strong variations about \pm 1 and small variations in the central part. The contribution of the first interval (containing -1 ) is given, for large $t-n$, by :

$$
\begin{aligned}
& \int_{-1}^{-1+4^{n-t}} \rho(x) A^{\prime}\left(f^{t-n} x\right)\left(f^{t-n}\right)^{\prime}(x) X(x) d x \\
\sim \quad & \frac{4^{t-n}}{\pi} \int_{0}^{4^{n-t}} \frac{A^{\prime}\left(f^{t-n}(-1+u)\right) X(-1+u)}{\sqrt{u}} d u
\end{aligned}
$$

where $x=-1+u$. If $A^{\prime}\left(f^{t-n}(-1+u)\right) X(-1+u)$ does not vanish about $u=0(x=-1)$ and has small variations on this interval, this contribution is $\sim 4^{t-n} \int_{0}^{4^{n-t}} \frac{1}{\sqrt{u}} d u=2^{t-n}$, which diverges $\S$ when $t-n \rightarrow \infty$, with a rate exactly given by the pole $\lambda=\frac{1}{2}$. Therefore, another good benchmark of our method, in the case where the complex susceptibility has a pole in the upper-half plane, consists in computing (23), and taking the inverse Fourier series, providing in this way an approximation for $\Delta \rho_{t}(A)$. One should see then the exponential divergence with a rate $\log (2)$ (which is exactly the value of the Lyapunov exponent $\lambda$ ).

However, there is another effect that must be taken into account in the numerics. When the amplitude of the response, $\sim \epsilon 2^{t}$, is small one computes numerically the

$\S$ If one considers now the contribution of the "bulk" [inner intervals] to the integral (24) one can figure out that it does not diverge, essentially because the derivative $\left(f^{t-n}\right)^{\prime}(x)$ has alternating signs and because the density $\rho$ has small variations in the bulk. More precisely, the contribution of the bulk is provided by a decomposition on the Ruelle-Pollicott eigenfunctions $\sigma_{n}$ and decays exponentially due to mixing. Thus, the existence of a pole in the upper-half complex plane is due to a boundary effect obtained when the perturbation weights the points $\{-1,1\}$ (where the density diverges). 
first term of the Taylor expansion (19). But when $\epsilon 2^{t}$ becomes too large the numerical computation includes also the nonlinear terms and one computes in fact the complete susceptibility, including nonlinear terms. This one does not diverge because the trajectories of the perturbed system remain in the interval $[-1,1]$, but it does not depend linearly on $\epsilon$.

Therefore, one expects the following. There is a time cut-off $t_{m}(\epsilon) \sim-\frac{\log (\epsilon)}{\log (2)}$ beyond which one does not compute the linear response but the complete response, including nonlinear effects which saturate the growth of the perturbation. Then one should observe an exponential growth $2^{t}$ up to $t_{m}(\epsilon)$ in the linear response of the form $2^{t} H_{\left[0, t_{m}(\epsilon)\right]}(t)$, where $H_{I}()$ is the characteristic function of the interval $I$. The corresponding susceptibility is:

$$
\sum_{n=0}^{t_{m}}(2 \lambda)^{n}=\frac{1-(2 \lambda)^{t_{m}}}{1-2 \lambda}
$$

which reads, using the frequency $\omega$ :

$$
+i \quad \frac{(1-2 \cos (\omega))\left(1-2^{t_{m}} \cos \left(\omega t_{m}\right)\right)+2^{t_{m}+1} \sin (\omega) \sin \left(\omega t_{m}\right)}{5-4 \cos (\omega)}
$$

Therefore, it exhibits oscillations due to the cut off $t_{m}$. For longer time, mixing should lead to an exponential decay of the response.

To check this we have first computed (23) for the perturbation (17) and for the observable $A(x)=x-\frac{x^{2}}{2}$ (the derivative of $A$ gives a maximal value for the term $A^{\prime}\left(f^{t-n}(-1+u)\right)$ in equation (24) $)$. The function $X_{-}$has been computed with the recursion (16) up to the order 4. The $\epsilon$ values $10^{-2}, 10^{-3}, 10^{-4}\left(T=10^{5}, N=\right.$ 400), $10^{-5}\left(T=10^{6}, N=400\right), 10^{-6}\left(T=4 \times 10^{6}, N=1600\right)$ have been considered (but only $10^{-4}, 10^{-5}, 10^{-6}$ are represented in Fig. 2 a,b for the legibility of the figure.). We have used a fit procedure to compare the experimental data with the form (25). We have also computed the approximate linear response. The results are presented in Fig. $2 \mathrm{a}, \mathrm{b}$.

One observes indeed a clear dependency with $\epsilon$. Moreover, the real and imaginary part exhibit the expected oscillations due to the cut-off, with a perfect agreement with the form (25). Taking the inverse Fourier series one obtains the linear response in Fig. 2b. One sees clearly the exponential growth $2^{t}$ and the $\epsilon$ dependent cut-off. Performing a fit in $\log$ scale one obtains (fig. 3) an exponential increase with a rate 0.65 very close to the expect value $\log (2)=0.693$. After this there is an exponential decay with an approximate rate -0.24 . (We have only represented the fit for $\epsilon=10^{-6}$ but one sees easily in Fig 3 that the decay rate is similar for $\epsilon=10^{-4}, 10^{-5}$ ). This rate is slower than the first Ruelle Pollicott resonance $-\log (4)=-1.386$. It might be that we are observing a crossover regime where exponential amplification and exponential mixing are competing. 

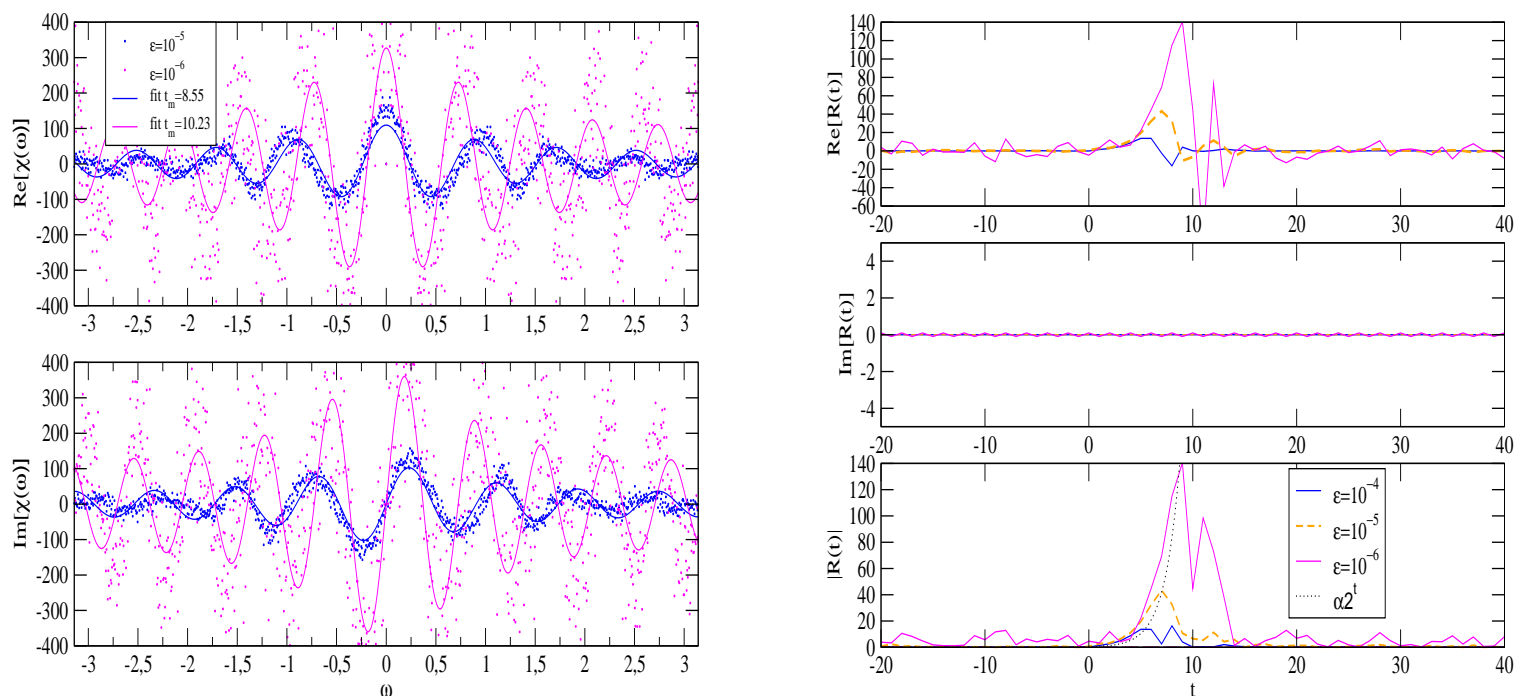

Figure 2. (a) Susceptibility for the perturbation $X_{-}(x)$, where $w$ was computed up to order 4 , and observable $A(x)=x-\frac{x^{2}}{2}$. The parameter $\epsilon$ takes the value $10^{-5}, 10^{-6}$. In full line are drawn the fitting curves obtained from (25). (b)Linear response.

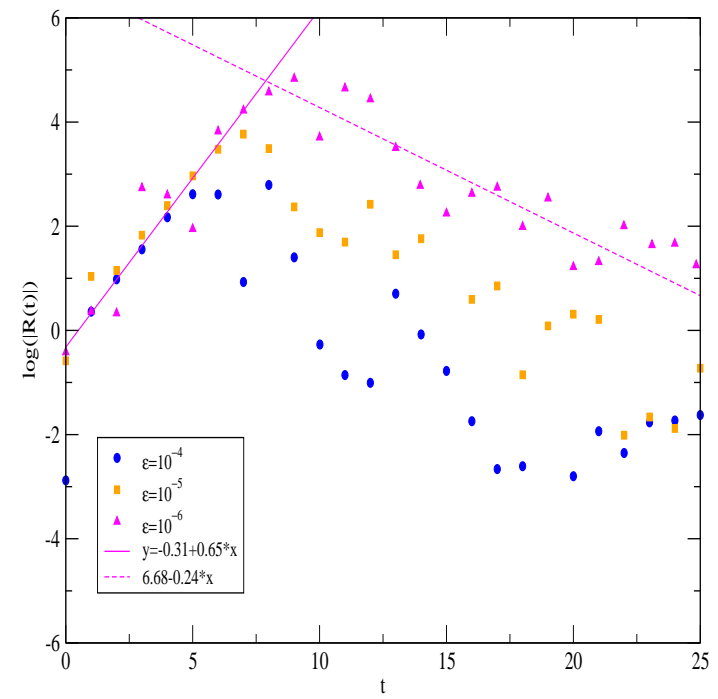

Figure 3. Fit of the linear response corresponding to Fig. 2b.

\section{The Hénon map.}

The Hénon map:

$$
\left\{\begin{array}{ccc}
x_{t+1} & = & 1-a x_{t}^{2}+y_{t} \\
y_{t+1} & = & b x_{t}
\end{array}\right.
$$

with $a=1.4, b=0.3$ has a strange attractor with a fractal structure. Moreover, numerical estimates of the largest Lyapunov exponent, using e.g. Eckmann-Ruelle 
algorithm [6] gives a value $\sim 0.422$. But the Hénon map is not uniformly hyperbolic. There are points with tangencies between stable and unstable manifolds. These points may be responsible for phenomena analogous to those described in the previous section, for specific perturbations weighting those points. From this observation, it is conjectured that there may also exist a pole in the upper-half complex plane (and possibly more complex singularities [20]). However, there is no mathematical result for this and the form of the perturbations/observable leading to such a singularity is not known.

Using the same method as in the previous section, a natural empirical approach consists in applying a perturbation $\epsilon e^{i \omega t}$ in one of the directions $(x, y)$ and investigating the effect on the linear response for one of the variables $(x, y)$. As an example, we have numerically computed the response of the observable $A(x, y)=x$ to a perturbation $\epsilon e^{i \omega t}$ in the direction $y$. Denote by $\chi_{x y}(\omega)$ and $R_{x y}(t)$ the corresponding susceptibility and approximate response. They are drawn in Fig. 4a,b respectively, for $\epsilon=10^{-3}\left(T=10^{5}, N=100\right), 10^{-4}\left(T=10^{5}, N=100\right), 2 \times 10^{-5}\left(T=10^{6}, N=\right.$ 400), $10^{-5}\left(T=10^{6}, N=1600\right)$. Note that essentially the same curves are obtained, up to a phase factor, when perturbing direction $y$ and computing the response of $y$. In particular $\left|\chi_{x y}(\omega)\right|=\left|\chi_{y y}(\omega)\right|$.
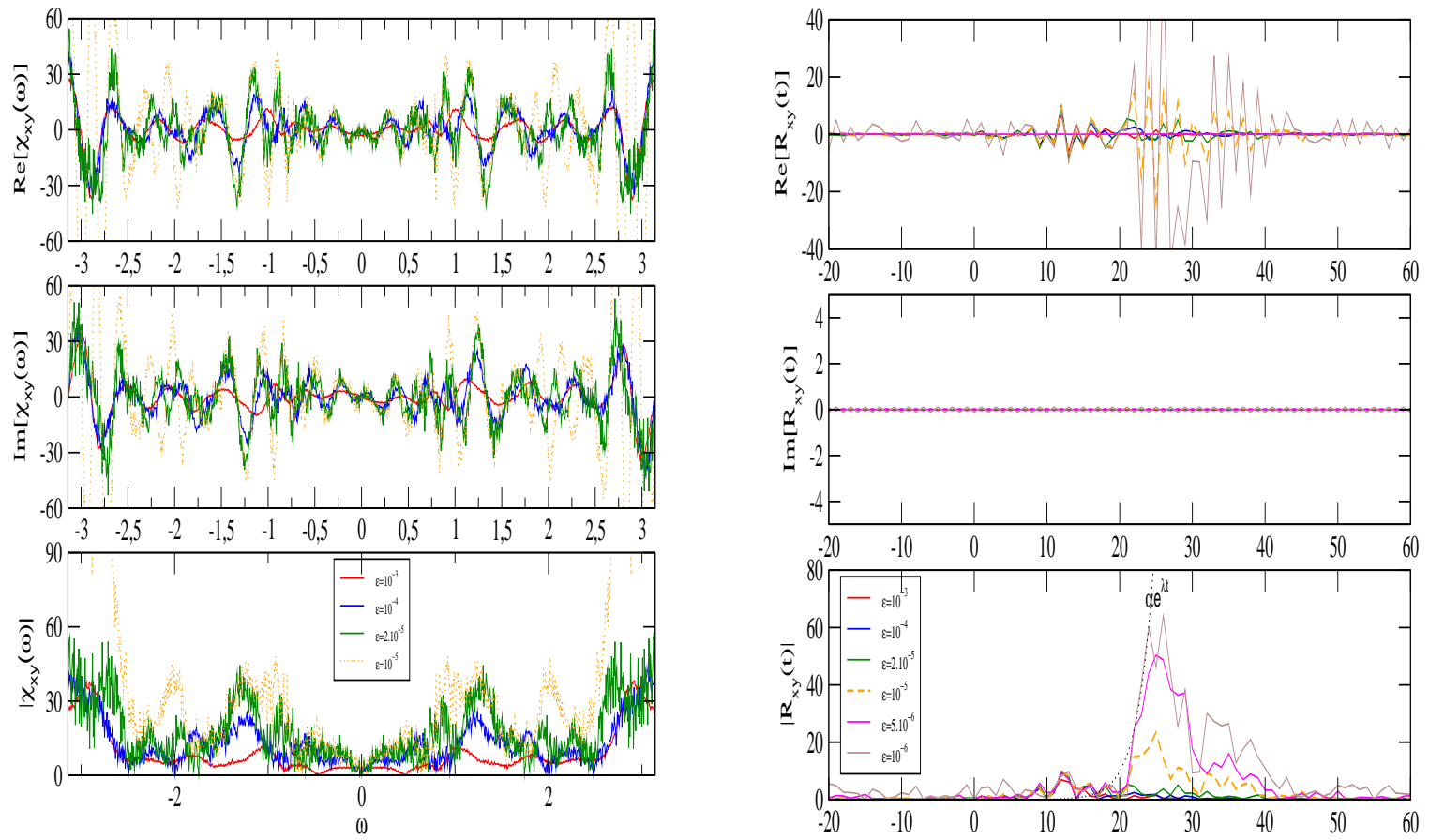

Figure 4. (a) Susceptibility for a constant perturbation $X=\epsilon$ in the direction $y$ for the observable $A(x, y)=x$. (b) Corresponding linear response.

One observes similar effects as in the previous section. The amplitude of the complex susceptibility increases with $\epsilon$ (and this effect is not stabilized, neither by increasing the time $T$ of average well beyond the criterion $\epsilon \omega T>>1$, nor by increasing 
the number of sample trajectories). The linear response has three parts. For short times $(t<20)$ there is a small bump for the modulus of the response: its height does not seem to depend on $\epsilon$. The perturbation/observables have a nonzero projection onto the Ruelle-Pollicott modes. It might be that this bump comes from this part, having a well behaved linear response. The second part exhibits an exponential increase with a time cut-off depending on $\epsilon$. This is very similar to the observations made for the logistic map: exponential amplification of the linear response until nonlinearities induce saturation of this effect. Then, mixing leads to an exponential decay (third part of the curve).

The exponential instability is well fitted by the curve $e^{\alpha} e^{\lambda t}$ with $\alpha=-6.80 \pm 0.87$ and $\lambda=0.455 \pm 0.038$ (see Fig. 5). Note that the positive Lyapunov exponent of the Hénon map is $0.42(2)$ for these values of $a, b$. The decay in the third part is well interpolated by a sum of exponential terms. The dominant term is $\beta e^{\gamma t}$ with $\gamma=-0.129 \pm 0.015$. The value of $\gamma$ is in agreement with the exponential decay of the correlation function $C_{x y}(t)=<f^{t}(x) y>-<x><y>$, where $<g>=\int g(X) \rho(d X), X=(x, y)$ and $\rho$ is the SRB measure ( Fig. 5). Thus the last part apparently obeys the fluctuation-dissipation theorem. Correlation function has been computed by the standard Fast Fourier Transform method (see [11], chapter 13.2).

Let us also remark that there are no thin peak in the susceptibility (the frequency resolution is 0.00612) and the thickness of the peaks does not change with $\epsilon$. Since the width $\|$ of the resonance peak is given by the imaginary part of the pole, this suggests that the imaginary part of the poles is bounded away from zero. This leads us to conjecture that there is no pole on the real axis (for the frequency $\omega$ ). This conjecture is compatibl@ with the available results (in particular exponential decay of correlations for many parameters $a$ and $b$ (see [4, 5, 23, 22])).

\section{Conclusion.}

There is currently an intensive research activity in mathematics dealing with linear response theory for interval maps (see e.g. [1, 2] and reference therein). In the case of non uniformly hyperbolic maps one can find examples where the susceptibility has an arbitrary large number of poles in the upper-half complex plane [21] or where the average of a smooth function with respect to the SRB measure of the perturbed system $f+\epsilon X$ is not even Lipschitz at $\epsilon=0$ [1]. These results are intriguing for a physicist and raise some natural questions: can we measure some effects related to these situations? What is the "genericity" of these examples? Do these effects arise in dynamical systems

$\|$ Consider the complex susceptibility in the neighbourhood of a simple pole $\omega_{0}=x_{0}+i y_{0}$. Then $\chi(\omega) \sim \frac{A}{\omega-\omega_{0}}$. Assume that this pole is close to the real axis. Set $\omega=x+i y$. On the real axis $|\chi(\omega)|^{2} \sim \frac{|A|^{2}}{\left(x-x_{0}\right)^{2}+y_{0}^{2}}$. The modulus is maximal at $x=x_{0}$, and has value $\frac{|A|}{y_{0}}$ (resonance peak). The width of the resonance peak is $2\left|x_{1}-x_{0}\right|$ where $x_{1}$ is such that $\left[\chi\left(x_{1}\right) \mid=\frac{|A|}{2 y_{0}}\right.$. Thus, the width is $2 y_{0}$. I I thank one of the reviewers for this remark. 

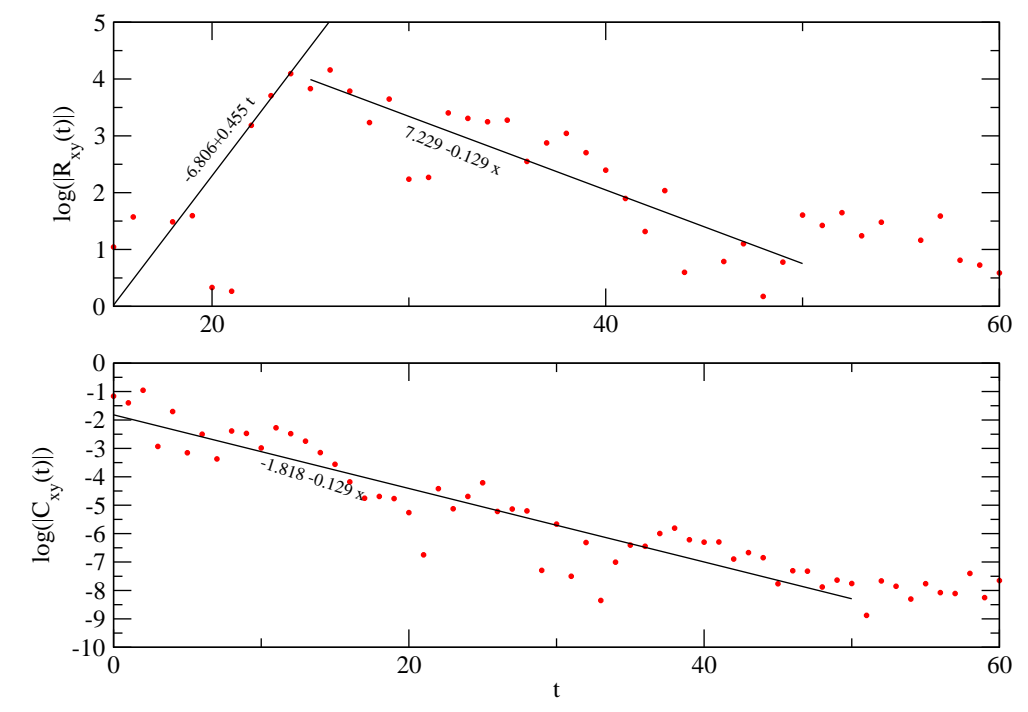

Figure 5. (a) Fit of the linear response $\left|R_{x y}(t)\right|, \epsilon=10^{-6}$ for the Hénon map. (b) Fit of the correlation function $C_{x y}(t)$ for the Hénon map.

modeling physical systems ? do they occur in larger dimensions? Answering these questions at the level of rigor of mathematics is probably a too formidable task at the current state of research in this field. One hope is that some hints can be provided by numerical approaches.

In this spirit, we have presented in this work a numerical procedure allowing to compute the complex susceptibility/linear response. For this, we use an approximation of the complex susceptibility (7) by a finite sum, which can be easily computed. In the cases where the series (7) converges this sum gives a fairly good approximation. When there is a pole in the upper-half plane a correct treatment of this sum allows us to recover the pole location. Applying this procedure to the Hénon model we conjecture that such a pole could also be present.

There are several possible developments of the present work. On mathematical grounds it could be interesting to apply this procedure to the cases discussed in e.g. [21, 1, 2] that can be addressed by rigorous methods. (In particular the cases in [1, 2] should provide examples where the linear response does not decay exponentially, but does not grow exponentially either.) An intermediate case between these mathematically tractable cases and the difficult Hénon model would be Collet-Eckmann maps. Another question is: "what is there beyond the pole" ? In some sense, the pole in the upperhalf complex plane may "hide" more complex singularities lying behind it such as cuts. Is it possible to have any (numerical) idea of which type of singularities are there ? A natural way of doing this is to remove the exponential instability by multiplying the perturbation by a damping factor (or equivalently to use a complex frequency $\omega$ ). This is natural from a mathematical point of view, but tricky in the numerics, because the damping factor is either smaller than the exponential instability (and the response 
grows rapidly inducing nonlinear effects, as we saw) or it is bigger (and the perturbation becomes rapidly numerically 0 , then we are measuring short time transients). One has thus to make small variations of the damping factor around the pole and look at the changes in the susceptibility/response curve.

On more physical grounds, a natural continuation of the present work, could be to consider a lattice of coupled logistic maps, to apply an harmonic perturbation at some point and look at the induced effects, in the spirit of this work. This would be one step towards the investigation the effects of large dimension ("thermodynamic limit") on the singularity induced by the microscopic dynamics.

\section{Acknowledgments}

I warmly thank David Ruelle for suggesting to me the present work and for helpful remarks and suggestions. I thank Jean-Louis Meunier for illuminating advices and Jacques-Alexandre Sepulchre for helpful discussions. I think that this paper has been widely improved due to the remarks of the referees and their positive criticism. I greatly acknowledge them.

[1] Baladi V., "On the susceptibility function of piecewise expanding interval maps", Preprint arxiv.org (v1: 2006, v2, v3:2007), to appear in Comm. Math. Phys. (2007).

[2] Baladi V., Smana D., "Linear response formula for piecewise expanding maps", Preprint arxiv.org, submitted for publication, (2007).

[3] Baladi V., Rugh H. H., Jiang Y., "Dynamical determinants via dynamical conjugacies for postcritically finite polynomials", J. Stat. Phys., 108, 973-993 (2002)

[4] Benedicks M. Young L.S., "Sinai-Bowen-Ruelle measures for certain Henon maps", Invent. Math, 112, (1993), 541-576;

[5] Benedicks M. Young L.S., "Markov extensions and decay of correlations for certain Henon maps", Asterisque 261, (2000), 13-56.

[6] Eckmann J.P., Ruelle D., "Ergodic Theory of Strange attractors" Rev. of Mod. Physics, 57, $617,(1985)$.

[7] Cessac B., Sepulchre J.A., "Stable resonances and signal propagation in a chaotic network of coupled units", Phys. Rev. E 70, 056111 (2004).

[8] Cessac B., Sepulchre J.A., "Transmitting a signal by amplitude modulation in a chaotic network"', Chaos 16, 013104 (2006).

[9] Cessac B., Sepulchre J.A., "Linear response, susceptibility and resonances in chaotic toy models", Physica D, Volume 225, Issue 1 , Pages 13-28, (2007).

[10] Gaspard P. "Chaos, scattering and statistical mechanics", Cambridge Non-Linear Science series 9, (1998)

[11] Press W, Flannery P., Teukolsky S., Vetterling W., "Numerical Recipes in C", Cambridge University Press (1988)

[12] Pollicott M., Invent. Math., 81, 413-426 (1985), Ruelle D., J. Differential Geometry, 25 (1987), 99-116.

[13] Reick C.H., "Linear response of the Lorentz system", Phys. Rev. E, 66, 036103, (2002).

[14] Ruelle D., J. Differential Geometry, 25 (1987), 99-116.

[15] D. Ruelle, Differentiation of SRB states, Com. Math. Phys. 187 (1997) 227-241.

[16] D. Ruelle, Differentiation of SRB states: Corrections and Complements, Com. Math. Phys. 234 (2003) 185-190. 
[17] D. Ruelle, General linear response formula in statistical mechanics, and the fluctuation-dissipation theorem far from equilibrium, Phys. Lett. A 245 (1998) 220-224.

[18] Ruelle D., "Differentiating the absolutely continuous invariant measure of an interval map $f$ with respect to f", Commun. Math. Phys., 258, 445-453 (2005).

[19] D. Ruelle, Smooth Dynamics and new theoretical ideas in nonequilibrium statistical mechanics, $J$. of Stat. Phys. 95 (1999) 393-468.

[20] Ruelle D. Private communication.

[21] Jiang Y., Ruelle D., "Analyticity of the susceptibility function for unimodal Markovian maps of the interval." Nonlinearity 18, 2447-2453 (2005).

[22] Wang Q.D., Young L.S., "Strange attractors with one direction of instability", Commun. Math. Phys. 218, (2001), 1-97.

[23] Young L.S. "Statistical properties of dynamical systems with some hyperbolicity", Annals of Math., (1998), 585-650.

[24] Young L.S. "What are SRB measures and which dynamical systems have them ?", J. Stat. Phys., 108, (2002), 733-754. 